\title{
La politique de l'eau en Afrique du Sud : le difficile équilibre entre développement durable et valorisation optimale de la ressource
}

David Blanchon

\author{
(2) OpenEdition \\ Journals \\ Édition électronique \\ URL : http://journals.openedition.org/developpementdurable/1735 \\ DOI : 10.4000/developpementdurable.1735 \\ ISSN : $1772-9971$ \\ Éditeur \\ Association DD\&T
}

\section{Référence électronique}

David Blanchon, «La politique de l'eau en Afrique du Sud : le difficile équilibre entre développement durable et valorisation optimale de la ressource », Développement durable et territoires [En ligne],

Dossier 6 | 2006, mis en ligne le 10 février 2006, consulté le 21 décembre 2020. URL : http:// journals.openedition.org/developpementdurable/1735; DOI : https://doi.org/10.4000/

developpementdurable.1735

Ce document a été généré automatiquement le 21 décembre 2020.

\section{(c) (1) \&}

Développement Durable et Territoires est mis à disposition selon les termes de la licence Creative Commons Attribution - Pas d'Utilisation Commerciale 4.0 International. 


\title{
La politique de l'eau en Afrique du Sud : le difficile équilibre entre développement durable et valorisation optimale de la ressource
}

\author{
David Blanchon
}

1 Depuis les années 1970, le statut de l'eau a profondément évolué dans les pays en développement. Considérée comme un instrument d'aménagement du territoire à l'ère des grands barrages à buts multiples, l'eau est devenue, dans les politiques proposées par la Banque mondiale ou le Global Water Partnership, un bien économique qu'il faut valoriser et distribuer au mieux, en tenant compte des coûts et bénéfices induits par chaque aménagement. Le quatrième principe de gestion de l'eau adopté à la conférence de Dublin en 1992 affirme ainsi clairement : «Pour tous ses différents usages, souvent concurrents, l'eau a une valeur économique et, à ce titre, devrait être reconnue comme un bien économique. (...) La non-reconnaissance par le passé de la valeur économique de l'eau a conduit à des gaspillages et des usages dommageables pour l'environnement de la ressource. Gérer l'eau comme un bien économique est un bon moyen pour obtenir un usage efficient et équitable, et pour encourager la conservation et la protection des ressources $»^{1}$.

2 L'évolution de la politique de gestion de l'eau en Afrique du Sud semble apparemment s'inscrire dans ces deux phases. A partir de 1950, l'Afrique du Sud a entrepris la construction d'un grand système de transfert d'eau, «vitrine » du régime d'apartheid, pour mettre en valeur les terres semi-arides du sud et de l'ouest du pays. Puis, après l'arrivée au pouvoir de l'ANC en 1994, le nouveau régime a explicitement adopté les "principes de Dublin », dès le premier Livre Blanc exposant sa politique de l'eau (RSA, 1994, p. 7). 
3 Le passage d'une gestion de l'offre à une gestion de la demande doit théoriquement permettre à la fois de respecter l'environnement grâce à une utilisation plus parcimonieuse de la ressource et de soutenir la croissance économique, nécessaire à la réduction du chômage qui touche près de $40 \%$ de la population sud-africaine. Si elle est combinée avec des mécanismes de compensation sociale, la gestion de la demande peut également permettre de rattraper les inégalités passées en terme d'accès à l'eau. Ces objectifs, exposés dans le Livre Blanc sur la politique de l'eau de 1997 (RSA, 1997), forment le cadre idéologique de la nouvelle loi sud-africaine sur l'eau de 1998.

4 Plus qu'un bilan des politiques post-apartheid, dont les instruments sont à peine fonctionnels, nous avons choisi d'étudier dans cet article deux dynamiques paradoxales que nous avons constatées lors de nos différentes études sur le terrain ${ }^{2}$. La première est la poursuite de la construction de grands barrages et surtout la multiplication des nouveaux projets de transfert inter-bassins malgré la mise en place progressive et affichée de politiques de gestion de la demande, supposées rendre ces monstres techniques à terme inutiles. La seconde combine deux options apparemment antinomiques : il s'agit du projet de construire des barrages pour maintenir un débit réservé, théoriquement calqué sur l'écoulement naturel, pour protéger les derniers écosystèmes naturels des rivières sudafricaines.

5 L'étude de ces deux paradoxes soulève de nombreuses questions à propos des nouveaux modes de gestion de l'eau en Afrique du Sud : quelle est la portée réelle du changement de 1994 ? Que faire des barrages et des transferts légués par le régime précédent ? Comment satisfaire à la fois les trois exigences du développement durable, en matière sociale, environnementale et économique? Au-delà du discours sur le développement durable, quels sont les outils les plus efficaces pour le mettre en pratique, dans un pays «en transition" et "en développement"? Enfin, sur quelles structures sociales (ONG, gouvernements locaux, associations d'utilisateurs) s'appuyer pour porter ces réformes?

6 L'objet de cet article est d'apporter des éléments de réponse à ces interrogations à travers l'étude tout d'abord du rôle de l'eau dans la structuration de l'espace et du territoire sudafricain, puis des modalités de mise en place d'une nouvelle politique de l'eau, et enfin des convergences et contradictions entre la politique de développement durable affichée par le gouvernement et l'accélération de la concurrence entre territoires.

1. Espace hydraulique, territoire hydropolitique en Afrique du Sud

7 Par son inégale répartition, l'eau est un des principaux éléments structurants de l'espace sud-africain. De plus, la valorisation des ressources hydrauliques a été un outil majeur des politiques de manipulations du territoire ${ }^{3}$, parallèlement et parfois en contradiction avec les autres politiques spatiales menées par le régime d'apartheid ${ }^{4}$.

La discordance entre les ressources en eau et les centres de consommation

8 Le trait marquant de l'Afrique australe est l'opposition entre la façade ouest, longée par le courant de Benguela, et marquée par l'aridité, et la côte orientale, arrosée par les pluies de saison chaude (été austral). Le Grand Escarpement, haut de 2000 à 3500 mètres, qui serpente à moins de 250 kilomètres de l'Océan Indien, accentue la dichotomie. Sur le haut plateau intérieur, les précipitations ne sont plus que de 650 millimètres par an à hauteur de Johannesburg, et décroissent régulièrement vers l'ouest.

9 Cette opposition est renforcée par l'évolution de variabilité, qui, relativement faible à l'est, devient de plus en plus forte en allant vers l'Atlantique : autrement dit, plus l'on va vers l'ouest, plus les ressources sont faibles, et moins elles sont fiables. Le seul fleuve qui 
tempère cette répartition est l'Orange, qui descend des hautes montagnes du Lesotho: mais il n'a ni la puissance, ni la régularité qui pourraient en faire un «Nil » d'Afrique australe, tel que l'ont rêvé les premiers explorateurs de l'intérieur du continent.

La répartition des populations à la veille de la colonisation européenne recoupe en grande partie la configuration de l'espace hydraulique sud-africain. Les plus grandes densités de populations africaines se trouvaient essentiellement sur la façade orientale (KwazuluNatal et Cap de l'Est) ainsi qu'au nord du pays.

Mais, comme le montre la figure 1, les principaux centres de consommation en Afrique du Sud sont actuellement le plus souvent situés dans des régions peu dotées en eau.

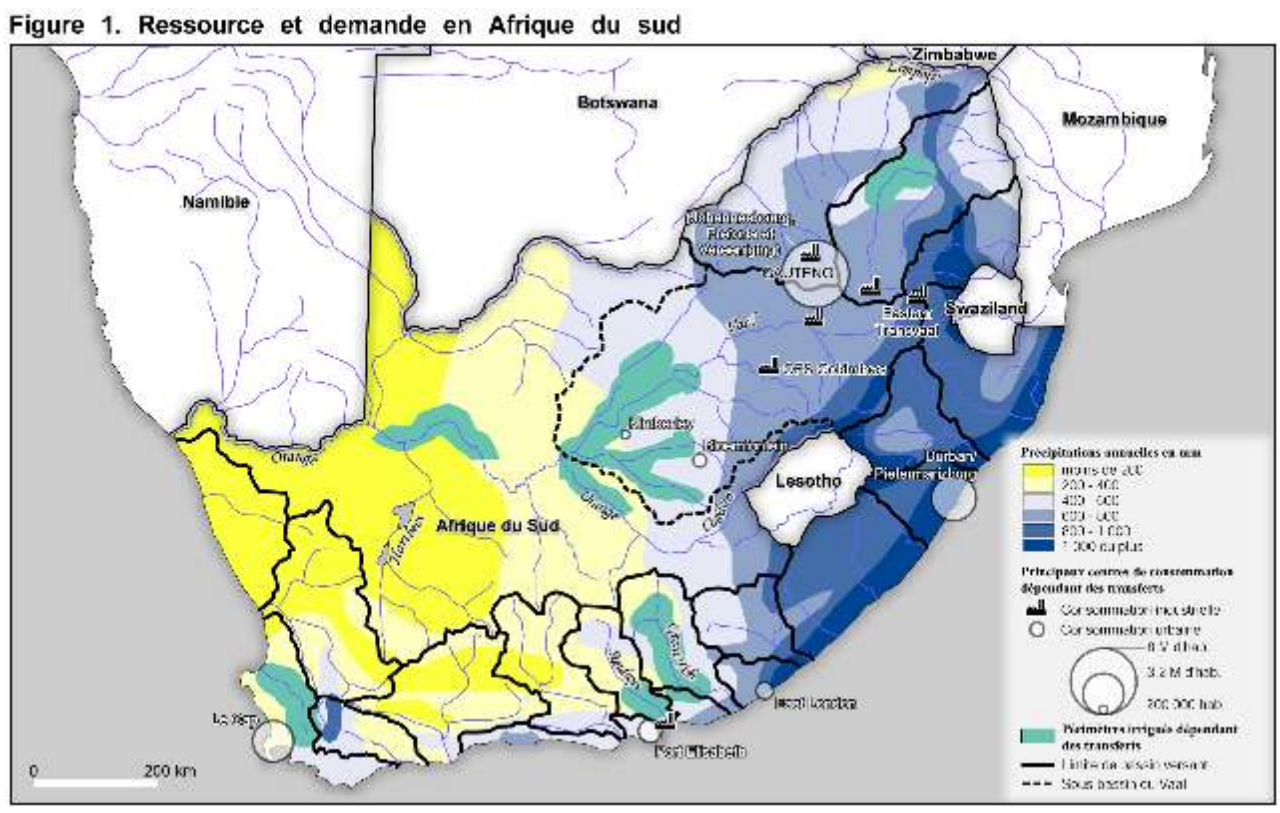

Source : divers documents du DWAF ; conception D. Blanchon ; réalisation D. Blanchon et unité de cartographie du Département de Géographie de I'Université de Laval (Québec)

[charger la figure docannexe.html?id=1819 ]

discordance entre les ressources en eau et les principaux centres de consommation s'explique en partie par les héritages des dynamiques de la colonisation. Le développement économique moderne du pays a été guidé, après l'arrivée des Hollandais, en 1652, par le commerce avec la métropole (port du Cap), puis, au XIX ${ }^{\text {ème }}$ siècle, par l'exploitation des mines de diamant (Kimberley) et d'or (Johannesburg). De même, l'agriculture commerciale des agriculteurs blancs a fait appel, dès le milieu du XIX ${ }^{\text {ème }}$ siècle, à l'irrigation, mobilisant des ressources de plus en plus importantes et lointaines.

Un choix capital : la politique de transferts d'eau

L'éloignement entre les ressources disponibles et les centres de consommation induit par la colonisation a fait naître très rapidement des tensions et des concurrences entre les territoires pour l'accès à l'eau. L'exemple le plus marquant est la ville de Johannesburg, qui se trouve exactement sur la ligne de partage des eaux entre les bassins versants de l'Orange et du Limpopo, et dont les ressources locales sont très faibles. La ville, née après la découverte de l'or en 1886, a dû commencer à pomper de l'eau dans le Vaal dès 1903. Un premier barrage a été construit en 1923, et, dès la fin dès années 1920, l'Etat sudafricain envisagea la construction d'un très grand barrage ${ }^{5}$, tant la concurrence avec les consommateurs situés en aval devenait forte. 

existaient : soit promouvoir un développement industriel et économique dans les régions les mieux dotées en eau, soit transférer l'eau sur plusieurs centaines de kilomètres vers les centres de développement existants. La première solution, proposée par le Directeur de l'Irrigation, L.A. McKenzie, en 1949, s'avérant trop difficile à mettre en œuvre ${ }^{6}$, c'est la deuxième qui a été privilégiée : quarante ans plus tard, la plupart des grands bassins versants sud-africains sont interconnectés, et plus de $4 \mathrm{~km}^{3}$ d'eau sont transférés par an, grâce à un système de régulation qui stocke $25 \mathrm{~km}^{3}$ d'eau, soit plus de $75 \%$ de la ressource utile (Figure 2).

Figure 2. Transferts d'eau on Afrique du Sud

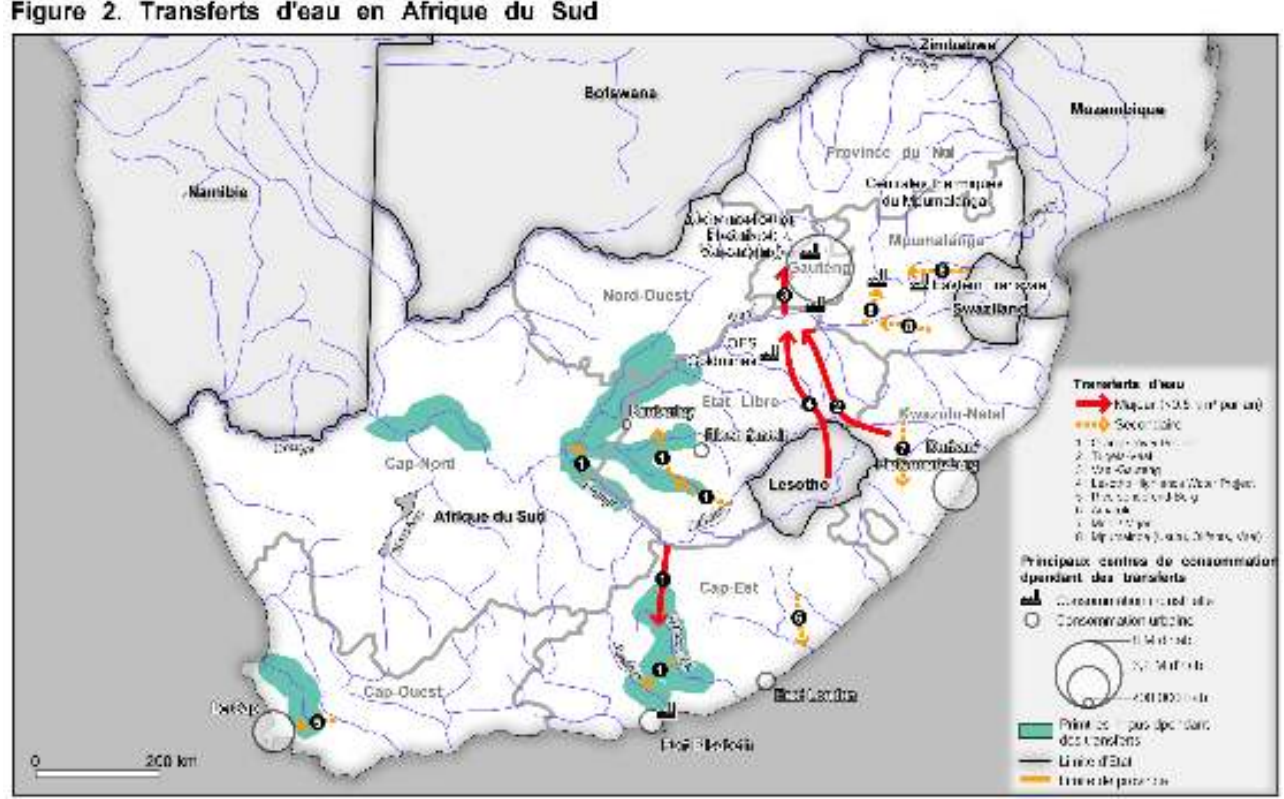

: divers documents du DWAF ; conception D. Blanchon ; réalisation D. Blanchon et unité de cartographie du Département de Géographie de l'Université de Laval (Québec)

Il n'est pas de notre propos de revenir ici sur l'histoire de ces transferts, ni sur les débats qui marquèrent sa construction. Des travaux récents ont montré que, même si l'on peut y trouver une logique a posteriori, il n'a jamais existé de plan d'ensemble (Blanchon et Turton, 2005). Chaque transfert a été mis en chantier selon sa logique propre, en fonction d'objectifs qui n'avaient que peu de rapport avec la politique hydraulique : l'Orange River Development Project (ORDP) a été lancé pour restaurer la confiance intérieure et extérieure $\mathrm{e}^{7}$ dans le régime d'apartheid après le massacre de Sharpeville de $1960^{8}$; la construction du Lesotho Highlands Water Project (LHWP) en 1986 est également liée à des considérations de politique internationale, car il permettait à l'Afrique du Sud, soumise à des sanctions, de contrôler directement le Lesotho et d'avoir accès aux financements internationaux par l'intermédiaire de ce pays (Blanchon, 2001).

19 Malgré leur diversité, tous les transferts répondent à une logique commune : apporter de l'eau de façon assurée aux divers usagers en utilisant l'espace pour contrer la variabilité temporelle des cours d'eau sud-africains. La nécessité de maintenir un débit assuré qui tend inexorablement à s'amenuiser est un des moteurs de l'extension toujours plus grande du réseau de transfert (Basson, 1994). Plus le système est étendu et maillé, moins une sécheresse localisée aura d'impact sur la production assurée du système global.

Les tensions du complexe hydropolitique sud-africain 
20 Le coût très important de ces systèmes a une conséquence majeure : comme l'écrit un auteur sud-africain, T. Basson (1994), « le système est conçu pour transporter de l'eau de là où elle est abondante vers là où l'on en a besoin; par conséquent, l'eau coule habituellement vers l'argent », ou, plus exactement, l'argent et le pouvoir.

21 Les transferts sont ainsi d'excellents indicateurs des tensions du territoire sud-africain et traduisent un rapport de force régional à un moment donné, qui explique leur direction. L'ORDP montre le pouvoir politique des agriculteurs des vallées de la Great Fish et de la Sundays dans les années 1960. C'est également la dernière et plus importante manifestation de la "mission hydraulique » de l'Etat sud-africain. L'objectif de faire fleurir le désert et la domination complète des ressources naturelles était alors un des fondements du régime d'apartheid, pendant lequel, de 1948 aux années 1970, les représentants des régions rurales (ou d'origine rurale) étaient prédominants au parlement.

23 La construction du LHWP, vingt-cinq ans plus tard, traduit en revanche le poids économique de la région de Johannesburg, seule capable de justifier et de supporter la charge financière des travaux.

24 L'extrême complexité du système sud-africain de transfert s'explique ainsi par les tensions entre le pouvoir politique, aux mains des électeurs blancs ruraux, et le pouvoir économique, concentré dans la métropole de Johannesburg.

La construction des transferts a eu trois conséquences principales. La première est la transformation des cours d'eau sud-africains en systèmes hybrides : avec la multiplication des transferts, les hydrosystèmes - organismes linéaires - sont devenus des réseaux maillés hybrides, où il est très difficile de distinguer le naturel de l'artificiel. Tout au plus peut-on y cerner des "gradients de naturalité », pour reprendre l'expression de J. Lecomte (1999).

26 Cette mutation a fait éclater le cadre du bassin versant, et induit une concurrence généralisée entre les territoires pour l'accès à l'eau. Tous les utilisateurs doivent alors justifier leur utilisation de l'eau, pour des motifs politiques ou économiques, de la ville de Johannesburg aux périmètres irrigués de l'Orange inférieur, en passant par les centrales thermiques du Mpumalanga ou encore la ville de Port Elisabeth. Il n'y a plus d'utilisateur « naturel » et incontestable de l'eau.

27 Ce double mouvement a enfin créé une polarisation politique forte des questions liées à la gestion de l'eau, avec l'opposition affichée de l'ANC de Nelson Mandela aux options choisies par le régime d'apartheid, principalement pour des motifs économiques - les barrages sont perçus comme des instruments du développement inégal - mais aussi, en collaboration avec des ONG «blanches", en prenant en compte des problématiques environnementales (Bond, 2002).

2. Le passage à la gestion de la demande : ruptures et continuités

L'aspect politique de la gestion de l'eau est apparent dès l'introduction du Livre Blanc de 1994 sur l'eau, le premier rédigé par le nouveau gouvernement de l'ANC, où il est écrit que « l'eau était essentiellement utilisée par un groupe dominant qui avait un accès privilégie à la terre et au pouvoir économique » et que « la victoire de la démocratie demandait que la politique nationale sur l'utilisation de l'eau et que la loi sur l'eau soit revue » (RSA, 1994). 

$1994^{9}$ et ont été inscrits dans la nouv
en 1956 par le régime d'apartheid. Un affichage révolutionnaire

L'esprit de la loi de 1998 a été exposé dans le Livre Blanc rédigé par les services du Department of Water Affairs and Forestry (DWAF) en 1997 (RSA, 1994). Les 21 propositions principales se résument en un slogan simple « un peu, pour tous, pour toujours » (Some, For All, For Ever), qui redéfinit complètement l'usage de l'eau, et par conséquent l'utilisation du territoire en Afrique du Sud. Ces trois axes s'inscrivent dans la perspective du développement durable (expression citée à vingt-trois reprises dans le Livre Blanc de 1994), dans ses dimensions environnementales, économiques et sociales.

31 Le premier («Some ») est l'acceptation du manque d'eau au plan national, conformément aux projections les plus fiables. Ce changement concerne surtout la population blanche, très grosse consommatrice d'eau urbaine, et l'agriculture commerciale.

Le deuxième principe du Livre Blanc « For all » est plus délicat et surtout plus coûteux à mettre en pratique. Sur le plan législatif, il se traduit par l'abolition des droits prioritaires des riverains, les Riparian Rights, qui donnaient aux agriculteurs blancs une mainmise presque totale sur les ressources en eau. Il rappelle surtout l'obligation de fournir de l'eau en quantité suffisante à toute la population.

Enfin, à long terme, le troisième point «For Ever » est la traduction directe du concept de durabilité. Il implique une gestion intégrée des hydrosystèmes, avec une plus forte intervention de l'Etat, et le renforcement des sanctions en cas de manquements à la législation sur l'eau. La traduction de ce principe est l'établissement d'une Reserve, débit inaliénable et prioritaire sur tous les autres usages. Elle est constituée de deux parties égales :

- Les besoins humains élémentaires pour la boisson, la préparation de la nourriture et l'hygiène personnelle, ce qui représente environ 25 litres par personne et par jour.

- La réserve dite environnementale soit « l'eau qui est nécessaire pour la protection des écosystèmes aquatiques «II est précisé que cela concerne autant la quantité que la qualité des eaux ${ }^{10}$.

Le changement de régime introduit également une nouvelle philosophie de gestion de l'eau qui ne repose plus sur l'augmentation continue de l'offre, mais sur la gestion de la demande qui devrait permettre une meilleure allocation de la ressource. La conséquence logique de cette politique est la fin des transferts d'eau inter-bassins, clairement stipulée dans le Livre Blanc de 1997, où il est écrit: «les transferts inter-bassins devront se conformer à des obligations particulières de planification. Celle-ci doit impliquer les institutions chargées de la gestion de l'eau des bassins receveurs et donneurs. Le bassin vers lequel l'eau sera transférée devra prouver que toute l'eau actuellement disponible dans ce bassin est effectivement utilisée de façon optimale et que des mesures efficaces pour l'économiser sont appliquées $»^{11}$.

Ce nouveau mode de gestion de l'eau politique est présenté comme l'exact contraire de la politique d'apartheid, résumée aux yeux de l'ANC par le slogan «All, For Some, Now ». Une pratique réformiste Mais si l'affichage est "révolutionnaire », la mise en œuvre de la nouvelle politique est très pragmatique et reprend des options déjà défendues au sein même du DWAF et soutenues par les instances internationales. 

un équilibre délicat entre d'une part la nécessaire amélioration du service - tant en quantité qu'en qualité - et d'autre part l'engagement pris de préserver et si possible restaurer un environnement très profondément dégradé. L'ampleur du problème est manifeste quand on sait qu'environ 12 à 14 millions de Sud-africains n'avaient pas un accès correct à une source d'eau potable en $1994^{15}$, alors que les ressources disponibles sont très limitées ${ }^{16}$, et qu'il faut de plus soutenir la compétitivité de l'agriculture et de l'industrie sud-africaine en procurant une eau peu chère.

nouvelle politique de l'eau est donc en fait un compromis fragile entre les exigences contradictoires de protection de l'environnement, compétitivité économique, et résorption des inégalités. Elle suppose que la gestion de la demande soit assez efficace pour freiner la consommation et ainsi permettre la mise en place de la Reserve (débit réservé), ce qui doit satisfaire les exigences des défenseurs de l'environnement. En contrepartie, le fait de délimiter clairement les besoins environnementaux doit laisser à son tour assez d'eau pour promouvoir le développement économique du pays. L'eau 
devient alors à la fois un bien public environnemental et une ressource à valoriser au mieux.

L'établissement de la Reserve écologique permet de circonscrire la part de l'écoulement. suffisante au maintien de ce qui est défini comme bien public environnemental. Mais audelà de cette Reserve, la consommation restante doit s'inscrire dans un strict calcul de rentabilité économique. C'est dans cette perspective que les services du DWAF ont calculé des indices de valorisation en terme de valeur ajoutée et d'emplois, non seulement par secteur économique (l'agriculture apparaît très défavorisée), mais aussi par régions : il en ressort que le Gauteng est de loin la région la mieux à même de valoriser l'eau en Afrique du Sud, avec un rapport de 1 à 244 en terme de valeur ajoutée et 1 à 80 en terme d'emplois par rapport à d'autres régions comme l'Orange inférieur (DWAF 1999).

Deux écueils menacent cependant ce compromis : le premier porte sur le choix et le « calibrage » des outils pour porter concrètement les nouvelles options politiques, que ce soit lors de la détermination du débit réservé que pour le calcul du prix de l'eau permettant à la fois un développement économique et la réallocation de la ressource pour les plus pauvres.

46 La seconde controverse porte sur les interprétations possibles du discours général du DWAF présenté depuis 1994. Il est ainsi concevable de faire une lecture « environnementaliste et sociale » du slogan « Some, For All, For Ever » qui signifierait la fin de la construction de barrages, la réallocation forcée des ressources de populations blanches dispendieuses vers les populations défavorisées ${ }^{17}$, ou au contraire d'adopter une position «développementaliste et libérale » en prônant la poursuite des transferts, la régulation de la demande par la politique des prix ${ }^{18}$, et, le cas échéant, si la croissance économique le demande, la construction de barrages et de transferts.

3. Protection de l'environnement et valorisation des ressources en eau : convergences et contradictions

47 La conjonction des deux controverses a rapidement mis fin au compromis de 1994 sur la politique sud-africaine de l'eau. Dans les débats, on retrouve alors le plus souvent les mêmes protagonistes, dont les positions avaient peu évolué depuis les années 1980, soit d'une part, les groupes écologistes comme la section locale International River Network ou des associations locales d'usagers à Johannesburg protestant contre la hausse future des prix, et de l'autre les cabinets de consultance chargés de la planification des grands systèmes de transferts (Bond, 2002).

La rupture du compromis de 1994

Le premier cas concret fut la décision de poursuivre le LHWP, largement décrié par l'ANC lors de la signature lançant le projet en 1986. Pourtant, K. Asmal, alors à la tête du DWAF, justifia ainsi sa décision : « Il y a dix ans, je m'opposais au traité sur le Lesotho Highlands Water Project (LHWP) pour des raisons politiques, et maintenant je dois assurer sa mise en service (...). Entre temps beaucoup de choses ont changé (...). L'Afrique du Sud a besoin d'eau pour son développement économique qui est si essentiel pour faire progresser le niveau de vie de notre peuple (...). Dans une région où le chômage est omniprésent, nous ne pouvons pas nous en tenir à un développement zéro au nom de la protection de l'environnement » (Asmal 1996).

Ce discours correspond au passage d'une conception de durabilité «forte", à une vision beaucoup plus large du développement durable, clairement exprimée depuis. M. Muller, directeur général du DWAF, écrivait ainsi en 2001 dans les colonnes du Mail and Guardian : 
«Les développements nécessaires sont menacés par des opinions (parfois délibérément) inexactes propagées par des critiques (...). Nous ne pouvons pas arrêter le développement dans le Tiers-Monde à cause des demandes de protection de l'environnement venant des pays riches qui ont déjà dégradé leur environnement (...). La réalité est que notre climat est aride et variable et que notre société est profondément divisée et inégalitaire $»^{19}$. Le discours du "développement durable" des ressources en eau est officiellement maintenu, mais avec un changement radical du contenu des politiques. La rupture du compromis entre les défenseurs de l'environnement et les partisans d'une augmentation de l'offre entrainna deux conséquences majeures, qui expliquent les paradoxes évoqués en introduction.

L'impossible débit réservé

51 La première conséquence est la subordination de la protection de l'environnement à des considérations économiques et sociales. Si la Reserve est un usage premier et inaliénable de l'eau, qui donne des droits aux cours d'eau au même titre qu'aux humains, elle doit alors être comprise comme la quantité d'eau nécessaire au maintien des fonctions des hydrosystèmes pour qu'ils continuent à être normalement exploitables et qu'ils ne nuisent pas à la santé des riverains.

C'est dans ce sens qu'a été construit le mode de calcul du débit réservé. Les premiers travaux pour calculer le débit réservé (Instream Flow Requirement) ont commencé dès 1980, en fixant le débit réservé pour maintenir les écosystèmes à $11 \%$ du débit naturel. Depuis, les méthodes de calcul ont été affinées pour prendre en compte les besoins effectifs de certains habitats, faire varier le débit réservé en fonction de débits constatés, et pour inclure partiellement la variabilité naturelle des cours d'eau sud-africains (Davis et Day, 1998).

Malgré ces progrès, les résultats obtenus, comme on le voit dans le cas du cours moyen de l'Orange (figure 3), sont très loin des valeurs observées depuis la construction des barrages ${ }^{20}$, et de toute façon sans aucun rapport avec les temporalités hydrologiques, marquées par la variabilité: suivre le rythme «naturel» impliquerait d'arrêter l'écoulement ou de provoquer de très fortes crues de façon aléatoire.

54 Ce calcul n'est donc en aucun cas fait pour rétablir le fonctionnement «naturel » des rivières, dont on ne sait de toutes façons que peu de choses. Il s'inscrit dans la continuité des politiques mises en place dans les années 1970 et se rattache en partie à des modes de gestion conservationnistes. Le but reste, comme l'écrivait A.D. Lewis, directeur du DWAF en 1928, qu' " aucune goutte ne se perde dans l'océan sans avoir été utilisée », dans ce cas précis pour maintenir des écosystèmes jugés remarquables. Ce raisonnement explique pourquoi il est alors tout à fait concevable de construire des barrages pour maintenir un débit réservé, comme c'est le cas dans le cours inférieur de l'Orange, où la construction d'un nouveau barrage, à 250 kilomètres de l'Océan Atlantique, pourrait permettre de gérer au mieux l'embouchure du fleuve protégée par une convention RAMSAR. 
Figurc 3. Débit mensucl moyen et Reserve pour l'Orange à Upington

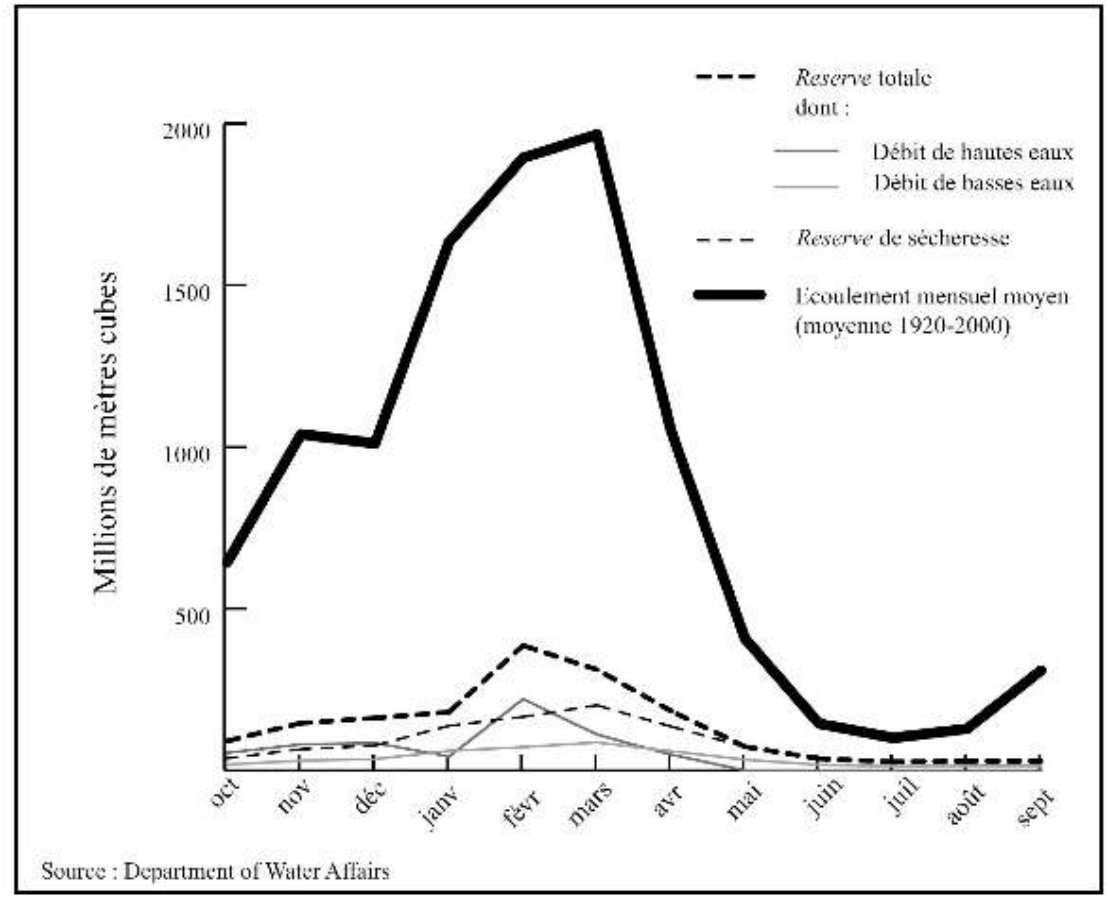

[charger la figure docannexe.html?id=1821]

Vers une concurrence généralisée pour l'eau entre les territoires

La deuxième conséquence de la rupture du compromis de 1994 découle de la première. L'établissement d'un débit réservé, parfois très bas, permet une utilisation et une valorisation libre des eaux « restantes ». La Reserve joue alors un rôle similaire à celui des transferts, qui, en brisant le cadre du bassin versant, « libèrent » également les eaux. Il en résulte une concurrence généralisée entre tous les utilisateurs et tous les territoires concernés, sur les cours d'eau donneurs et receveurs.

Le phénomène a commencé dès les années 1960, comme nous l'avons vu, mais il prend aujourd'hui de nouvelles formes. Tout d'abord avec l'apparition de nouveaux acteurs : les écosystèmes définis d'intérêt public, mais aussi les consommateurs pauvres et les agriculteurs africains et métis autrefois privés de l'accès à l'eau (emerging farmers). Ensuite par l'établissement de nouvelles règles, avec l'apparition de marchés de l'eau et la justification de l'utilisation de l'eau, par des critères économiques et secondairement sociaux. Enfin par la multiplication des échelles de jeu, avec, outre la concurrence exacerbée entre les régions, l'apparition de concurrences locales avec l'institution des marchés de l'eau, et l'apparition d'une échelle supérieure, par la valorisation de l'eau sur les marchés mondiaux dans le cadre d'échanges d'eau virtuelle.

De ce nouveau jeu concurrentiel, trois tendances émergent: la concentration vers Gauteng, la captation des ressources en eau par les villes au détriment des périmètres irrigués, et enfin, au niveau local, la concentration des ressources en eau des périmètres irrigués au bénéfice des grands producteurs (Armitage et al., 1999), sous le double effet de la libéralisation des échanges et de la création des marchés de l'eau.

La conjonction de ces facteurs explique le développement rapide de nouveaux projets de transfert, comme le montre la figure 4. L'introduction combinée de calculs de stricte rentabilité économique et de marchés locaux de l'eau (ou à défaut d'une politique du 
« juste prix » de l'eau) justifie la construction de nouveaux transferts vers les régions qui seront à même de valoriser au mieux l'eau, surtout si l'on estime que les besoins environnementaux de la rivière donatrice sont satisfaits par la Reserve. Cette logique explique comment la politique de gestion de la demande peut conduire à la multiplication des projets de transferts.

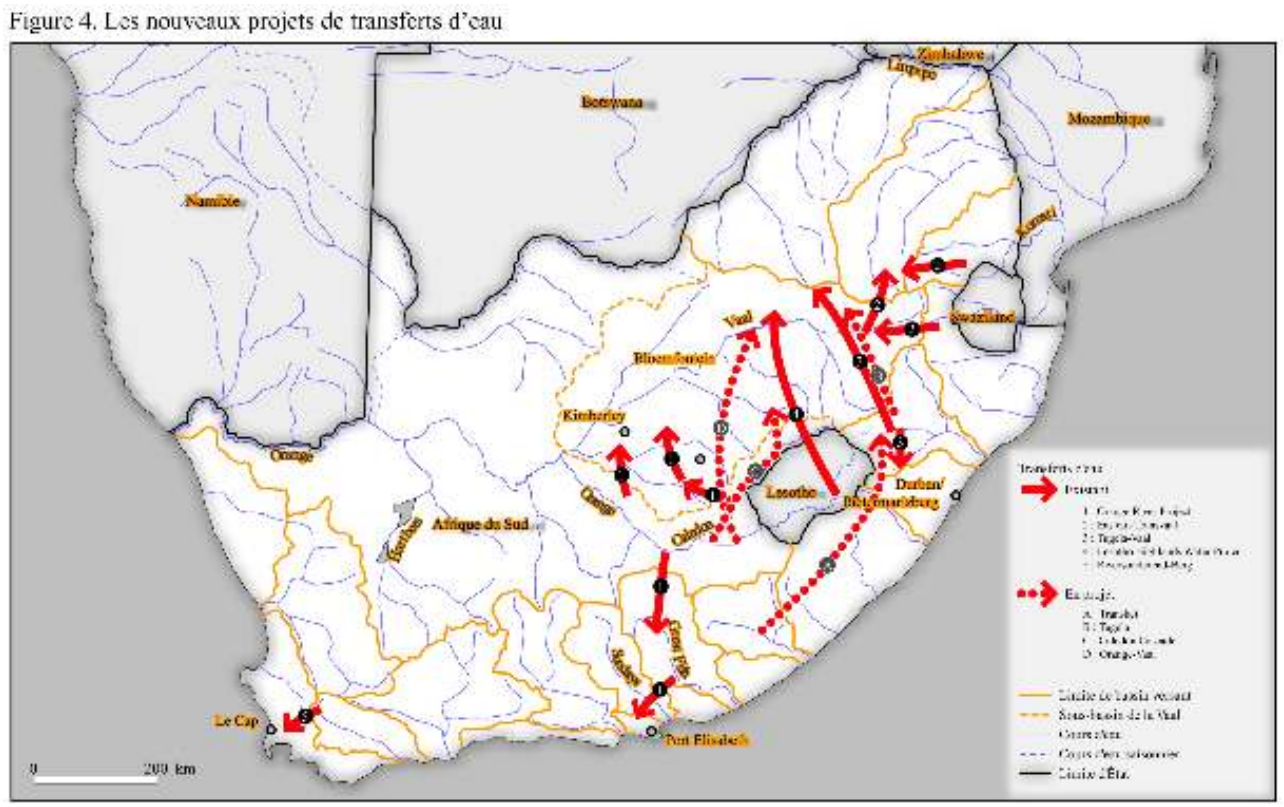

Source : divers documents du DWAF ; conception D. Blanchon ; réalisation D. Blanchon et unité de cartographie du Département de Géographie de I'Université de Laval (Québec)

[charger la figure docannexe.html?id=1822]

Conclusion

61 En définitive, l'exemple sud-africain montre que l'invocation du principe de développement durable ne permet qu'un compromis théorique fragile qui ne résiste pas aux premiers choix pratiques. C'est l'absence d'une définition claire des objectifs environnementaux, couplée à l'ambiguïté sur le statut des transferts et le décalage entre un affichage « révolutionnaire » et une pratique réformiste dans les politiques de l'eau, qui explique à la fois l'apparition de situations paradoxales que nous avons évoquées en introduction et l'accélération des concurrences pour l'eau.

La rupture du compromis esquissé en 1994 entre différents groupes (DWAF, associations d'usagers, ONG centrées sur le protection de l'environnement) est selon nous révélatrice du problème plus général du statut de l'eau dans les politiques environnementales. Toute action sur les hydrosystèmes ne porte pas sur des données naturelles, mais sur des constructions statistiques (pour la construction des barrages, qui repose sur des extrapolations à partir de débits partiellement et mal enregistrés, ou des prévisions de consommation), ou des déductions à partir d'analyses locales pour le calcul du débit réservé.

Les tableaux des ingénieurs hydrauliciens comme les états d'équilibre des géomorphologues et des biologistes comprennent donc à la fois et inséparablement des processus naturels et anthropiques. Parallèlement, comme l'écrit J. P. Bravard (1998), il est impossible de définir un «état originel " des écosystèmes et donc de délimiter l'impact d'une perturbation, comme la construction d'un barrage. Il y a donc plus une différence de "degré d'artificialité " que de nature entre le débit réservé et une 
canalisation en béton, ce qui explique que les ingénieurs sud-africains peuvent envisager de construire des barrages pour maintenir un débit réservé naturel.

Des trois pôles du développement durable (économique, écologique et social), l'environnement est, pour ces raisons, le plus fragile, car il se présente comme une donnée scientifique, alors qu'il inclut toujours des options sociales et politiques. C'est ce qui explique pourquoi, en Afrique du Sud, les préoccupations environnementales ont rapidement été minorées par rapport aux impératifs sociaux et économiques.

\section{BIBLIOGRAPHIE}

Armitage R.M., Nieuwoudt W.L., Backeberg G.R., 1999, « Establishing tradable water rights : case studies of two irrigation districts in South Africa », Water SA, vol. 25, n³, pp. 301-310.

Asmal K., 1996, « Keynote Address », In : Group for Environmental Monitoring, Lesotho Highlands Water Workshop-Record of Proceedings, Johannesburg, 29-30 August 1996.

Basson M.S., 1994, Probabilistic Management of Water Resource and Hydropower Systems, Highlands Ranch (Colo., USA), Water Resources Publications, 424 p.

Blonchon D., 2001, «Les nouveaux enjeux géopolitiques de l'eau en Afrique australe », Hérodote, $\mathrm{n}$ $\circ 102$, troisième trimestre, pp. 113-137.

Blanchon D., Turton A., 2005, « Les transferts d'eau en Afrique du Sud », In : Lasserre F. (Ed.), Les transferts massifs d'eau dans le monde, Presses de l'Université du Québec.

Bravard J.-P., 1998, « Le temps et l'espace dans les systèmes fluviaux, deux dimensions spécifiques de l'approche géomorphologique », Annales de Géographie, n599, pp. 3-15.

Bond P., 2002, Unsustainable South Africa : Environment, Development and Social Protest, University of Natal Press, Pietermaritzburg, 449 p.

Davies B.R., Day J.A., 1998, Vanishing Waters, Le Cap, University of Cape Town, 487 p.

Department of Water Affairs, 1986, Management of the Water Resources of the Republic of South Africa, Pretoria.

Department of Water Affairs, 1999, Orange River Development Project Replanning Study : Main Report, Pretoria, DWAF (DWAF Report n P D/000/006697).

Gervais-Lambony P., 1997, L'Afrique du Sud et les Etats voisins, Paris, Armand Colin/Masson, 254 p.

Kokot D.F., 1967, « Is water the limiting factor in the development of South Africa ? », Optima, 1967, vol. 17, pp. 12-17.

Lecomte J., 1999, « Réflexions sur la naturalité », Courrier de l'environnement de l'INRA, 37, pp. 6-10.

Mackenzie, L.A., 1949, « The Water Resources of the Union in Relation to the Development and Decentralisation of Industries ", Address delivered at the 32nd annual convention of the S. A. federated chamber of industries at Johannesburg.

Republic of South Africa, 1970, Report of the Commission on Enquiry on Water Matters, Pretoria, Government Printers. 
Republic of South Africa, 1994, White paper on Water Supply and Sanitation Policy, WP 94, Gov. Publisher, Cape Town.

Republic of South Africa, 1997, White paper on a National Water Policy for South Africa, WP 04- 97, Gov. Publisher, Pretoria.

Turton A., Henwood H. (Eds.), 2002, Hydropolitics in the Developing World : a southern African Perspective, Pretoria, Awiru, $269 \mathrm{p}$.

\section{NOTES}

1. Une version française, commentée et actualisée des "principes de Dublin " est disponible sur le site du Global Water Partnership : http://www.gwpforum.org/gwp/ library/Catalyzing_change_French.pdf.

2. Ces missions ont été menées de 1999 à 2003 dans le cadre d'un travail de thèse portant sur les impacts environnementaux et les enjeux territoriaux des transferts d'eau interbassin en Afrique du Sud.

3. Dans le contexte sud-africain, ce terme désigne la politique dite de " grand apartheid", c'est-à-dire la constitution de Bantoustans indépendants ou autonomes et le déplacement massif de populations africaines, à la fois dans les villes et vers les Bantoustans dont ils étaient devenus « citoyens » (Gervais-Lambony, 1997).

4. Nous entendons par « espace hydraulique » la répartition naturelle des ressources, c'est-à-dire des précipitations et des transferts naturels par les grands hydrosystèmes ; par « territoire hydropolitique », l'exploitation de ces ressources, la production de l'eau brute par les barrages, le transport de l'eau par système de transfert, en enfin la valorisation des ressources. C'est une traduction possible du terme anglais "waterscape».

5. Ce barrage, le Vaal Dam, fut mis en service en 1937.

6. Selon ce plan, il s'agissait de promouvoir le développement industriel uniquement dans les zones côtières de l'Est, bien dotées en eau, qui présentaient de plus l'avantage de se trouver près des concentrations de populations africaines. Mais cela demandait l'arrêt de la croissance de la métropole de Johannesburg et une réorganisation du système de transport du pays, essentiellement pour les produits miniers (McKenzie, 1949).

7. Lors de l'inauguration du plus grand barrage du projet, le ministre de l'eau de l'époque, S.P Botha, déclara : «Le projet a été conçu comme un acte de foi à une époque où des attaques étaient menées contre nous à la fois de l'extérieur et de l'intérieur, et notre réalisation a convaincu même ceux qui sont contre nous que nous sommes déterminés à aller de l'avant ». Cité in Die Siviele Ingenieur in Suid Afrika, vol. 14, février 1972, pp. 31-32. 8. La police sud-africaine avait tiré sur des manifestants noirs pacifiques et tué au moins 180 personnes.

9. Il y est écrit que « toute personne a le droit à un environnement qui ne soit pas dangereux pour sa santé et son bien-être, et de bénéficier d'un environnement protégé, pour le bénéfice des générations présentes et à venir » et dans le même temps que « toute personne a le droit d'avoir accès à suffisamment d'eau. »: Bill of the Rights, Constitution of South Africa, section 24 et 27 (1)(b).

10. Cette partie correspond partiellement à ce que l'on appelle en France « débit réservé ».

11. RSA, 1997, point 6.6.3. 
12. Republic of South Africa (1970).

13. Department of Water Affairs (1986).

14. A l'époque Bantoustan indépendant, reconnu par la seule Afrique du Sud.

15. Plus de trois millions n'avaient toujours pas un accès correct à l'eau potable en 2001.

Source : Stat SA Census 2001.

16. Entre 1000 et $1200 \mathrm{~m}^{3}$ par personne et par an selon les estimations (en France la disponibilité est de $3300 \mathrm{~m}^{3} /$ personne /an).

17. Dans la municipalité de Johannesburg, le rapport de consommation en eau est de 1 à 7 entre les quartiers blancs les plus favorisés et les townships. En 1994, le prix payé par les forts consommateurs était très faible.

18. C'est dans ce sens qu'a été introduite une politique tarifaire différenciée : les six premiers mètres cubes consommés mensuellement par ménage sont gratuits, alors que la consommation au-delà de 20 mètres cubes est surtaxée.

19. Mike Muller, "Right to reply ", Weekly Mail and Guardian, march 23 to 29, 2001.

20. Les deux grands barrages, Gariep Dam $\left(5 \mathrm{~km}^{3}\right)$ et Vanderkloof $\left(1.3 \mathrm{~km}^{3}\right)$ ont été mis en service au milieu des années 1970 et régulent partiellement le débit du fleuve.

\section{RÉSUMÉS}

L'objet de cet article est de présenter les difficultés liées à l'adaptation du concept de développement durable en Afrique du Sud. D'une part, à cause des spécificités du mode de mise en valeur des ressources hydriques dans ce pays, fondé sur des transferts d'eau à grande échelle, mais aussi à cause de l'ambiguïté même du concept. Notre article montre que, au-delà de l'affichage sous le terme de "développement durable ", les politiques de gestion de l'eau mises en œuvre depuis 1994 n'ont que provisoirement freiné la construction de barrages et de transfert et ont au contraire accéléré la concurrence entre les territoires. Cela est dû au statut ambigu de l'environnement dans ce pays, où le rapport entre protection de la nature, développement économique et justice sociale a été profondément altéré par quarante années d'apartheid.

politique de l'eau, gestion de la demande, transferts d'eau inter-bassins, protection de l'environnement, aménagement du territoire, afrique du sud

This paper questions the sustainability of the post-apartheid water policy in South Africa. Even if they were partly successful in the process of taking into account environmental problems and providing basic water services to previously disadvantaged communities, these policies have raised new problems. This paper shows that the implementation of the environmental Reserve and the water demand management policy have paradoxically accelerated the competition between regions for the access to water resources, and only temporarily stopped the building of huge dams and inter-basin transfers. One major reason is the controversial status of the environment in South Africa, deeply disturbed by the apartheid regime, and still politically and socially problematic. 
INDEX

Keywords : environmental management, water policy, water demand management, inter-basin transfers, south africa

\section{AUTEUR}

\section{DAVID BLANCHON}

David Blanchon est Maître de Conférences en Géographie à l'Université de Bordeaux III. Il a récemment soutenu, à l'université de Paris $\mathrm{X}$, une thèse sur les enjeux territoriaux et les impacts environnementaux des transferts d'eau inter-bassins en Afrique du Sud. 